\title{
Plasmapheresis Center
}

National Cancer Institute

\section{Source}

National Cancer Institute. Plasmapheresis Center. NCI Thesaurus. Code C133325.

A facility licensed by the FDA/CBER that collects source plasma or therapeutic exchange plasma for commercial distribution. 